\title{
Uso da biomembrana de látex em cavidade exenterada: relato de caso
}

\author{
The use of latex biomembrane in exenteration: case report
}

\author{
FernandoFugimoto ${ }^{1}$ \\ Ana Carolina Jervásio ${ }^{2}$ \\ Alexandre Kazuo Misawa ${ }^{3}$ \\ Renato Garcia ${ }^{4}$ \\ Suzana Matayoshi ${ }^{5}$
}

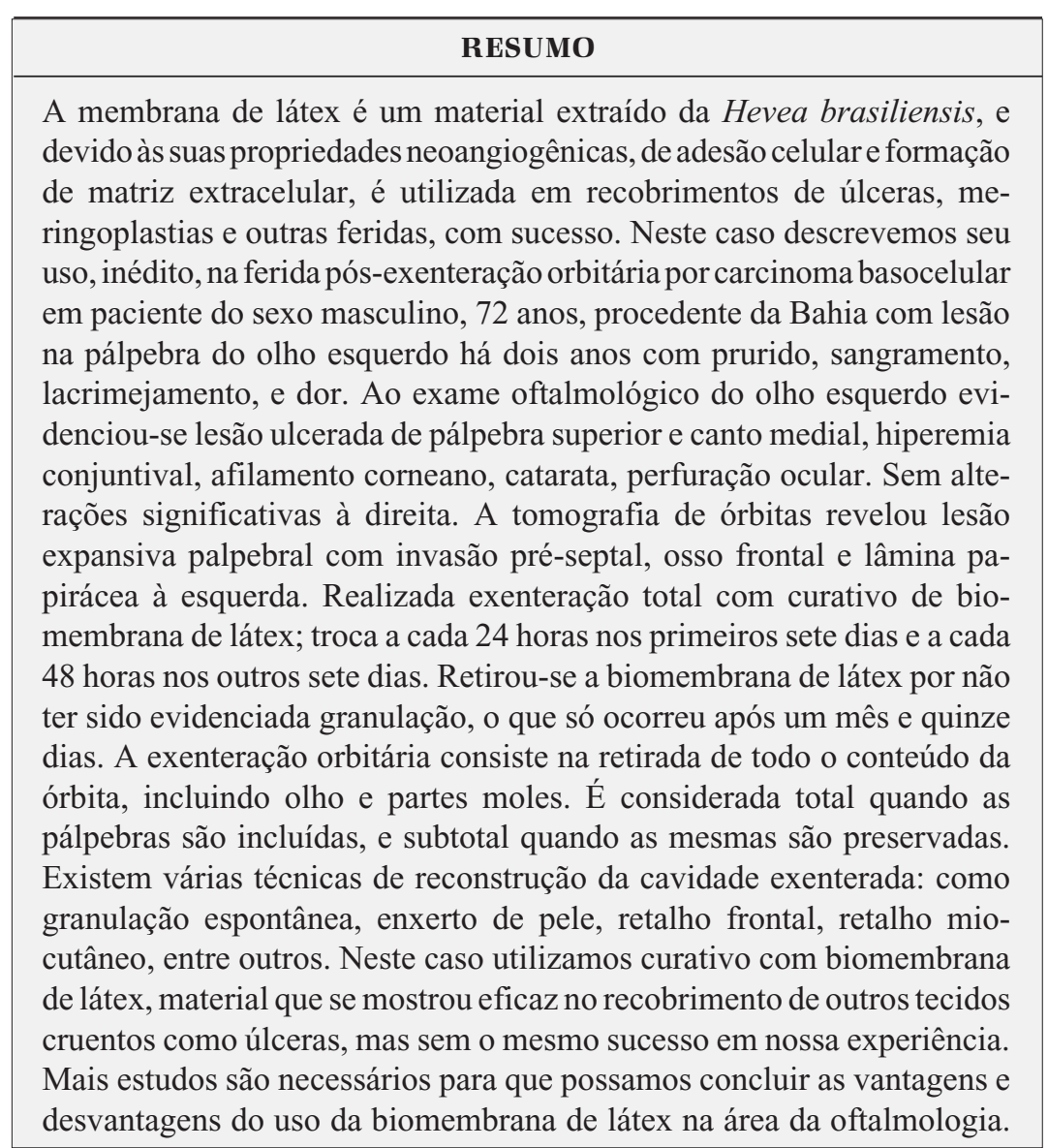

Descritores: Látex/uso terapêutico; Exenteração orbitária; Neoplasias palpebrais; Hevea; Relatos de casos [Tipo de publicação]

\section{INTRODUCÃO}

Neste trabalho apresentamos o uso inédito da biomembrana de látex na reepitelização da cavidade orbitária pós-exenteração. A biomembrana, produzida do látex extraído da seringueira Hevea brasiliensis pode ser usada em reparo de defeito parcial de esôfago, ressecções extensas de parede abdominal, substituição parcial de pericárdio, meringoplastias e reconstrução conjuntival; e também na cicatrização de feridas crônicas, tais como: úlceras por insuficiência vascular, diabética, de pressão, vasculogênicas, feridas cirúrgicas e traumáticas limpas ou infectadas ${ }^{(1-2)}$.

A biomembrana apresenta aumento da permeabilidade e fluxo vascular quando banhada com polilisina. Acredita-se que tenha como proprieda- 
des: atividade neoangiogênica, promoção de adesão celular e formação de matriz extracelular; e como principal vantagem menor risco de transmissão de doenças em relação aos materiais provenientes de tecidos animais. Embora potencialmente alergênica ${ }^{(3)}$, não há descrição de reação alérgica em casos $\operatorname{tratados}^{(1)}$.

O sucesso da aplicação dessa biomembrana na regeneração de tecidos encorajou-nos a estudar seu emprego na reconstrução da cavidade orbitária e a fixar como objetivo deste trabalho a verificação dos seus efeitos no processo de granulação desta cavidade.

\section{RELATO DO CASO}

Paciente de 72 anos, masculino, branco, procedente da Bahia com queixa de lesão na pálpebra superior do olho esquerdo há 2 anos acompanhado de prurido, sangramento, lacrimejamento e dor no olho esquerdo. Foi internado e encaminhado para o serviço de Plástica Ocular do Hospital das Clínicas para investigação diagnóstica.

Ao exame o paciente apresentava lesões eritematosas queratóticas nos membros superiores, múltiplos comedos gigantes no dorso, nódulo perláceo com telangiectasia na região malar à direita e placa perlácea no dorso nasal estendendo para pálpebras à esquerda. Foi encaminhado para o serviço de Dermatologia do Hospital das Clínicas que fez a hipótese diagnóstica de carcinoma basocelular na região malar, nasal e nas pálpebras à esquerda.

A acuidade visual para longe com correção era de 20/40 no olho direito e percepção de luz no olho esquerdo. No exame biomicroscópico do olho direito observou-se catarata nuclear $2+/ 4+$ e subcapsular $1+/ 4+$ e no olho esquerdo evidenciou-se lesão ulcerada da pálpebra superior e canto medial (Figura 1), hiperemia conjuntival, afilamento corneano, catarata nuclear

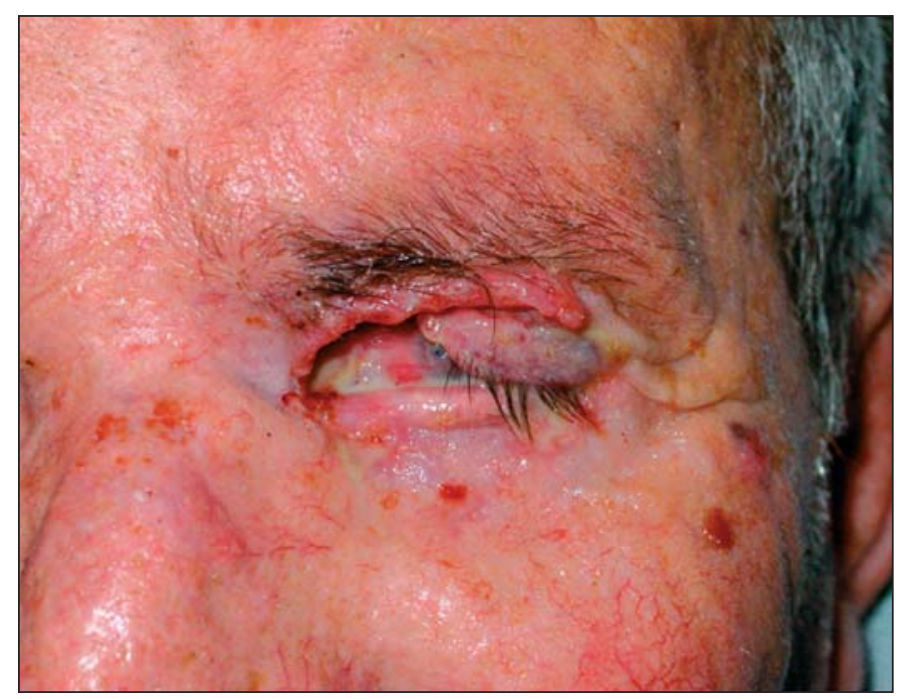

Figura 1 - Lesão expansiva ulcerada da pálpebra superior do olho esquerdo
2+/4+ e perfuração ocular na região perilimbar nasal tamponada pela íris medindo aproximadamente $1 \times 1 \mathrm{~mm}$. A pressão intra-ocular pelo tonômetro de Goldmann do olho direito era de $12 \mathrm{mmHg}$ e do olho esquerdo não foi mensurada devido à perfuração ocular. A fundoscopia do olho direito era normal. O esquerdo era de difícil avaliação devido à perfuração ocular.

Foi solicitada tomografia de órbitas que evidenciou lesão expansiva acometendo pálpebra superior do olho esquerdo em íntimo contato com o globo ocular medindo cerca de $1,8 \times 1,4$ $\mathrm{x} 1,4 \mathrm{~cm}$, gordura intra e extra-conais preservadas, globos oculares tópicos, nervos ópticos e musculatura ocular extrínseca preservadas, lesão expansiva palpebral com invasão pré-septal em contato com o globo ocular e invasão do osso frontal e lâmina papirácea à esquerda (Figura 2).

Realizada exenteração do olho esquerdo com ressecção da lâmina papirácea e parte do osso frontal com avaliação de margens cutâneas livres de tumor pelo exame de congelação no intra-operatório. Feito curativo com biomembrana de látex (Biocure ${ }^{\circledR}$ Pele Nova ${ }^{\circledR}$ ) para estimular granulação da cavidade orbitária com troca de curativo a cada $24 \mathrm{~h}$ nos primeiros 7 dias e a cada 48 h nos 7 dias subseqüentes (Figura 3A). A cada troca de curativo foi colhida cultura da secreção da cavidade e da biomembrana, e lavagem da cavidade com gentamicina. O resultado das culturas foi negativo. Como não foi evidenciado melhora na granulação da cavidade com a biomembrana de látex, foi optado por retirá-la e deixar a cavidade granular por segunda intenção, o que ocorreu após um mês e quinze dias (Figura 3B). O paciente foi encaminhado para adaptação de prótese ocular na cavidade orbitária à esquerda acoplada com óculos.

O resultado da biópsia da lesão malar evidenciou carcinoma basocelular esclerodermiforme, sendo feita sua exérese pelo serviço de Dermatologia do Hospital das Clínicas da FMUSP com reconstrução de retalho bilobado. No momento o paciente faz acompanhamento no setor de Plástica Ocular e de Dermatologia do Hospital das Clínicas.

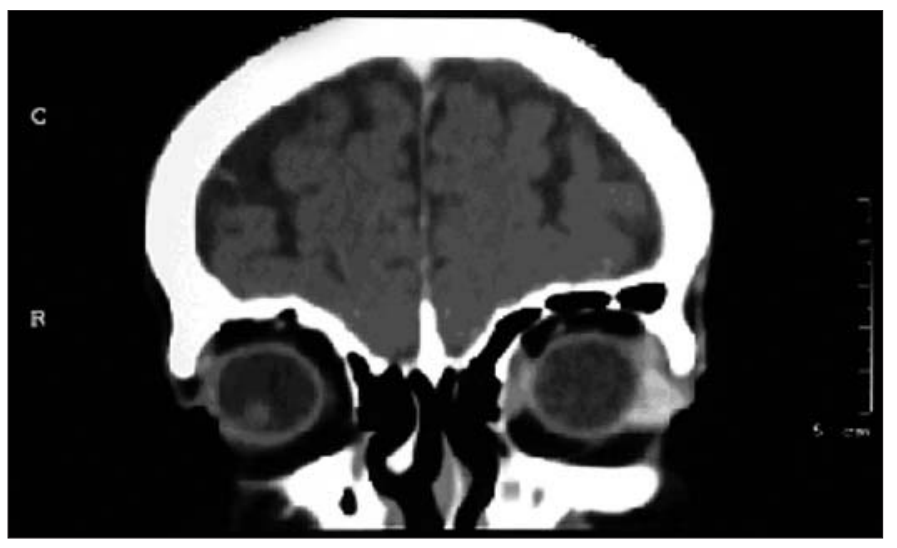

Figura 2 - Tomografia de órbita: lesão expansiva medindo cerca de 1,8 $\times 1,4 \times 1,4 \mathrm{~cm}$, gordura intra e extra-conais preservadas, nervos ópticos e musculatura ocular extrínseca preservadas, lesão expansiva palpebral com invasão pré-septal em contato com o globo ocular e invasão do osso frontal e lâmina papirácea à esquerda 

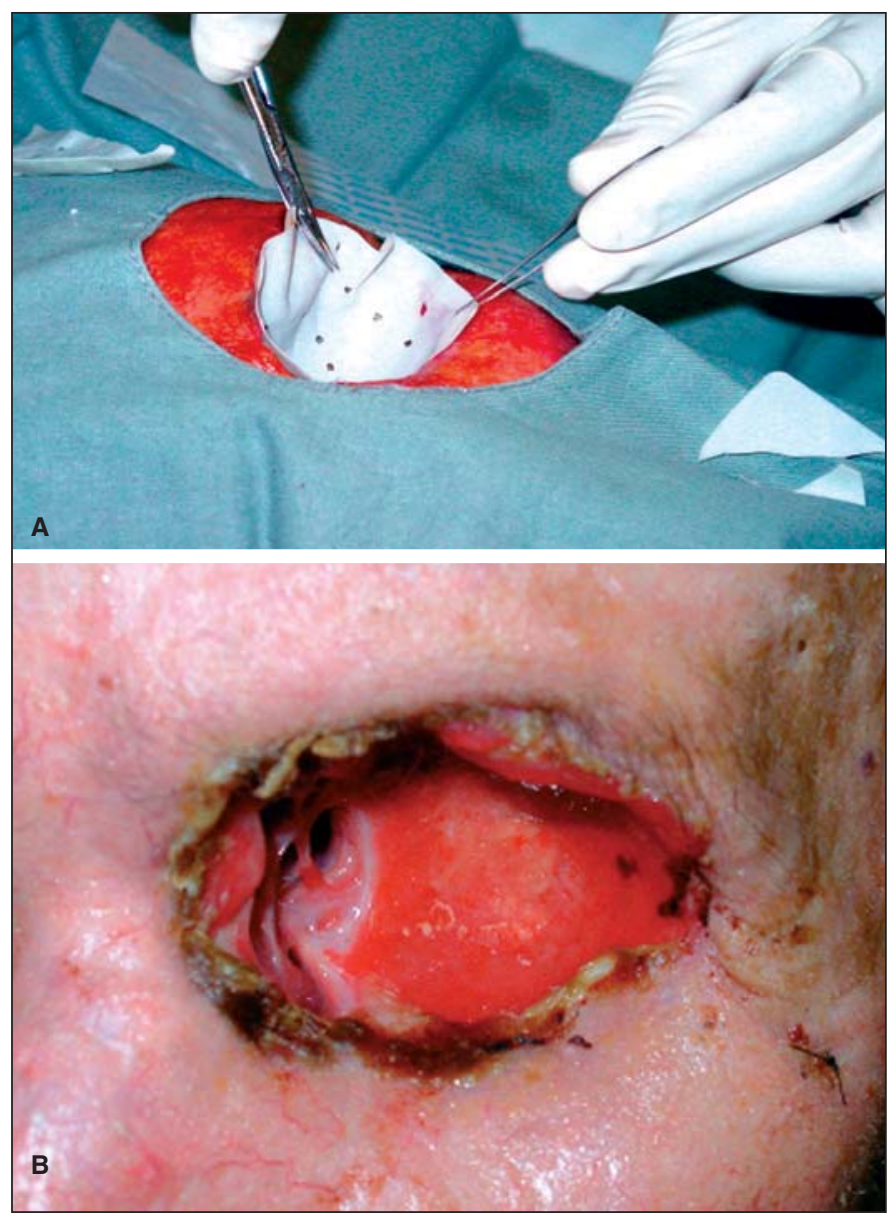

Figura 3- A) Curativo com biomembrana de látex (Biocure ${ }^{\circledR}$ Pele Nova ${ }^{\circledR}$ ) para estimular granulação da cavidade orbitária; B) Um mês e quinze dias de pós-operatório (granulação da cavidade orbitária)

\section{DISCUSSÃO}

A exenteração orbitária consiste no esvaziamento do conteúdo da cavidade orbitária, isto é, na extirpação do globo ocular e das partes moles. Está indicada em casos de tumores e ocasionalmente em infecções fúngicas periorbitárias ${ }^{(4)}$.

Dois procedimentos podem ser realizados: a exenteração subtotal (conservação parcial ou total das pálpebras) ou a completa (retirada de todo o conteúdo da órbita, inclusive pálpebras $)^{(4-5)}$. As pálpebras podem ser mantidas em casos de tumores primários da órbita ou extensão orbitária de tumores malignos da úvea ou conjuntiva, não ocorrendo em casos de tumores malignos primários das pálpebras ou da glândula lacrimal ${ }^{(6)}$. As técnicas de reconstrução da cavidade orbitária pós exenteração já descritas são: granulação espontânea, enxerto de pele de espessura parcial, retalho frontal, "flap" miocutâneo, implante de metil-metacrilato, enxerto dermogorduroso e enxerto muscular miocutâneo ${ }^{(4-5)}$. O uso de espuma de silicone moldável à órbita, foi descrito com resultados muito satisfatórios ${ }^{(7)}$.

A técnica de granulação espontânea apresenta como van- tagens a facilidade de execução, evita obtenção de retalhos cutâneos e permite como resultado final uma órbita mais rasa e de melhor adaptação de prótese. As desvantagens são em relação ao tempo de cicatrização mais longo e a necessidade freqüente de troca de curativos $^{(8)}$.

O enxerto de pele é o mais popular e é obtido principalmente da coxa. A principal desvantagem é a descamação de queratina na cavidade orbitária ${ }^{(9)}$.

$\mathrm{O}$ enxerto dermatogorduroso apresenta como principais vantagens a vascularização rápida e apresenta-se como superfície perfeita para crescimento e proliferação de células epiteliais vizinhas. As principais áreas doadoras são a coxa, o braço e o abdome ${ }^{(9-10)}$.

Há relatos de uso de "flap" miocutâneo do trapézio, deltotemporal, temporal e latissímo do dorso com pedículo vascular que faz anastomose com vasos faciais ${ }^{(11)}$. Apresenta como desvantagem a possibilidade de mascarar recorrência de tu$\operatorname{mor}^{(9)}$.

Neste caso, utilizamos a biomembrana de látex para estimular a cicatrização da cavidade orbitária, pois em tratamentos de úlcera por flebite crônica permitiu melhora na vascularização e capacidade de indução angiogênica e conseqüentemente a reepitelização ${ }^{(2)}$.

$\mathrm{O}$ uso da biomembrana na reconstrução conjuntival em olhos de coelhos foi satisfatória em $60 \%$ dos casos e os com esclera nua em apenas $20 \%{ }^{(1)}$. Isto sugere que a biomembrana de látex, à semelhança do que ocorre em outros tecidos, também promova a aceleração da cicatrização na conjuntiva. Outro fato relevante foi que o número médio de vasos por campo óptico na ferida cirúrgica dos olhos com biomembrana foi o dobro dos nos olhos com esclera nua. Isso vem reforçar as suposições da literatura de que a biomembrana realmente tenha propriedades neoangiogênicas. Uma vez que o processo de reparo tecidual não ocorre sem neoangiogênese, que a vasculatura neoformada corresponde a sessenta por cento de todo o tecido de reparo, e que as células caliciformes dependem de aporte sangüíneo para a sua manutenção, a membrana de látex parece promover condições biológicas satisfatórias para a recuperação conjuntival ${ }^{(1)}$.

Neste caso a granulação da cavidade orbitária com o uso da biomembrana de látex não foi satisfatória. Não sabemos se este resultado foi devido à grande área para que ocorresse a granulação ou se a biomembrana não promove a mesma. É o primeiro caso relatado do uso da biomembrana na tentativa de agilizar a granulação. Mais estudos e um maior número de casos são necessários para que possamos concluir as vantagens e desvantagens do uso da biomembrana na área da oftalmologia.

\section{CONCLUSÕES}

A biomembrana de látex é cada vez mais utilizada na reconstrução de áreas cruentas e mostra grande eficácia. Neste caso não obtivemos os resultados esperados o que nos deve 
impulsionar a estudar mais o uso deste material no meio oftalmológico.

\section{ABSTRACT}

The latex biomembrane is a material from Hevea brasiliensis and has angiogenic properties, cellular adhesion and extracellular matrix formation. It has been used for the treatment of ulcers in the inferior extremities caused by diabetes, chronic vascular insufficiency and timpanic perforations. In this study we report a case of biomembrane use to promote granulation in the orbital cavity after exenteration in a male, 72 years, who came into our service with a left eyelid lesion for two years. The ocular examination revealed a hyperemic conjunctiva, corneal thinning, cataract and ocular perforation in the left eye. The right eye had no alterations. There was an expansive and ulcerated lesion with orbital septum, frontal bone and lamina papiracea invasion confirmed by tomography. A total exenteration of the cavity was made and the biomembrane was applied to the affected site. Changes were made every 24 hours in the first seven days, and every other day in the following seven days. The biomembrane had to be taken from the patient because no granulation could be seen. Just one occurred and a half month later. Exenteration is a procedure in which all of the orbital content is removed. If the eyelids are included it is called total exenteration. There are many exenteration techniques as spontaneous granulation, skin grafts, miocutaneous flap, etc. In this case we used a material which has been successfully applied for other wounds, but with no efficiency in our experience. More studies are necessary so that we can present benefits to our patients in the ophthalmologic area with the latex biomembrane.

Keywords: Latex/therapeutic use; Orbit evisceration; Eyelid neoplasms; Hevea; Case reports [ Publication type]

\section{REFERÊNCIAS}

1. Pinho ECCM, Sousa SJF, Schaud F, Lachat JJ, Coutinho-Netto J. Uso experimental da biomembrana de látex na reconstrução conjuntival. Arq Bras Oftalmol. 2004;67(1):27-32

2. Frade MA, Valverde RV, de Assis RV, Coutinho-Netto J, Foss NT. Chronic phlebopatic cutaneous ulcer: a therapeutic proposal. Int J Dermatol. 2001;40 (3):237-40.

3. Muguerza J, Capo C, Porri F, Jacob JL, Mege JL, Vervloet D. Latex allergy: allergen identification in Hevea braziliensis fractions by immunoblotting. Clin Exp Allergy. 1996;26(10):1177-81.

4. Leite Filho M, Matayoshi S, Gonçalves JOR. Exenteração. In: Soares EJC, Moura EM, Gonçalves JOR. Cirurgia plástica ocular. São Paulo: Roca; 1997. p.447-56.

5. Matayoshi S. Exenteração. In: Matayoshi S, Forno EA, Moura EM. Manual de cirurgia plástica ocular. São Paulo: Roca; 2004. p.305-12.

6. Shields JA, Shields CL, Suvarnamani C, Tantisira M, Shah P. Orbital exenteration with eyelid sparing: indications, technique, and results. Ophtalmic Surg. 1991;22(5):292-7.

7. Benson MT, Gilmour H, Nelson ME, Rennie IG. Silastic foam dressing for healing exenteration cavities. Ophthalmic Surg. 1990;21(12):849-51.

8. Putterman AM. Orbital exenteration with spontaneous granulation. Arch Ophthalmol. 1986;104(1):139-40.

9. Mauriello JA Jr, Han KH, Wolfe R. Use of autogenous split-thickness dermal graft for reconstruction of the lining of the exenterated orbit. Am J Ophthalmol. 1985;100(3):465-7.

10. Shore JW, Burks R, Leone CR Jr, McCord CD Jr. Dermis-fat graft for orbital reconstruction after subtotal exenteration. Am J Ophthalmol. 1986;102(2):228-36.

11. Donahue PJ, Liston SL, Falconer DP, Manlove JC. Reconstruction of orbital exenteration cavities. The use of the latissimus dorsi myocutaneous free flap. Arch Ophthtalmol. 1989;107(11):1681-3. 